funded by Arthritis Research UK to support the administration and management of the internship programme. J. Adams: None. C. Bowen: None.

\title{
030 TRAINING THE NEXT GENERATION OF CLINICAL RHEUMATOLOGY RESEARCHERS: EVALUATION OF A GRADUATE ALLIED HEALTH PROFESSIONAL AND NURSE INTERNSHIP PROGRAMME
}

David Wright ${ }^{1}$, Mary Fry ${ }^{1}$, Jo Adams ${ }^{1}$ and Catherine Bowen ${ }^{1}$ ${ }^{1}$ School of Health Sciences, University of Southampton, Southampton, UNITED KINGDOM

Background: Building research capacity is an essential part of sustaining evidence based practice in nursing and allied health professions working within rheumatology. Whilst medical and dental professions have a strong tradition of research capacity building, the situation for allied health Professionals and nursing in the UK is less developed. This presentation reports on the interim findings of an evaluation of a collaborative internship programme across five UK universities from 2015-18. The internship included an eight-week programme of structured training workshops and research project delivery, and ongoing mentoring by experienced researchers. Sixteen interns were recruited from across the UK, including physiotherapists (7), podiatrists (5), occupational therapists (2) and nurses (2).

Methods: The evaluation employed mixed methods including: analysis of research metrics, an annual evaluation questionnaire sent to all 16 interns, and qualitative email interviews (8 interns, 9 mentors) conducted at the end of the internship programme. Interpretive phenomenological analysis of transcripts was used to identify recurring themes.

Results: Early quantitative outcomes from internship projects include three peer-reviewed publications, and 13 conference abstract presentations. Interns reported positive changes in their perceptions of research and rheumatology, including a realization that clinical academic pathways were possible. Skills attained of most value to interns were technical research (e.g. qualitative research), research process (e.g. securing funding), rheumatology knowledge (e.g. fatigue), and general skills (e.g. communication). Two domains of impact were identified. First, the programme directly impacted on research careers with four interns securing clinical academic positions and most others reporting commitment to pursuing active research in the near future. Second, the internship had an impact on practice for those entering full-time clinical careers. Interns spoke of their ability to be 'critically aware', seeking an evidence base for clinical decisions. Many spoke of a new confidence in expressing opinions with clinical colleagues. Others spoke of the need for patient-centered care, learned from the Patient and Public nvolvement training provided by the internship. Similarly, interns reported an increased awareness of the wider relevance of rheumatology, which influenced their subsequent clinical practice. One challenge for the internship programme identified by mentors was the difficulty in attracting interns from all Allied Health Professionals and Nursing backgrounds (notably nurses). In addition, several interns entering fulltime clinical roles reported difficulties in continuing research in environments that devalued such activity.

Conclusion: The collaborative internship programme has been successful in supporting research capacity building by introducing newly qualified allied health professionals and nurses to research and rheumatology. This has generated tangible benefits through research outputs, clinical academic careers and influencing clinical practice. The programme serves as a model for research capacity building for other health conditions and professions.

Disclosures: D. Wright: Other; DW received funds from Arthritis Research UK to undertake the evaluation. M. Fry: Other; MF was 